Ophthalmologe 2013 · 110:1132-1133

DOI 10.1007/s00347-013-2945-4

Online publiziert: 7. Dezember 2013

(c) Springer-Verlag Berlin Heidelberg 2013

\section{Diestelhorst}

Klinik und Poliklinik für allgemeine Augenheilkunde, Uniklinik Köln

\title{
Medikamentöse Therapie und Verlaufskontrolle der Glaukome
}

\section{Möglichkeiten und Grenzen der Lokaltherapie}

Liebe Kolleginnen und Kollegen, im vorliegenden Band möchten wir den aktuellen Stand der medikamentösen Therapie der Offenwinkelglaukome (OWG) fortsetzen. Sie haben sicher eine systematische Einteilung nach Pharmagruppen vermisst. Und auch die lokalen Nebenwirkungen sollen in ihrer Tragweite beleuchtet werden.

Eine Schematisierung der medikamentösen Glaukomtherapie ist zwar bezüglich der pharmakologischen Eigenschaften und Nebenwirkungen der jeweiligen Medikamente sinnvoll, im Praxisalltag bleibt die Flexibilität des Therapeuten gefragt.

Ziel jedweder Glaukomtherapie ist die Stabilität von Papille und Gesichtsfeld, unabhängig vom jeweiligen Augeninnendruck (IOD). Ziel der medikamentösen Lokaltherapie ist die konstante pharmakologische Senkung des IODs über $24 \mathrm{~h}$, 365 Tage, Jahre.

\section{\) Ziel jeder Glaukomtherapie ist die Stabilität von Papille und Gesichtsfeld}

Die Adhärenz/Nonadhärenz bestimmt den Erfolg/Misserfolg der Lokaltherapie. Eine maximale Lokaltherapie ( $>2$ Flaschen) ist nur selten über Jahre erfolgreich. Dies liegt an der mangelnden Adhärenz der Patienten (wir kommen später darauf zurück), die mit den Nebenwirkungen und den unzureichenden Appli- kationsmethoden der Augentropfen zusammenhängt.

Eine andauernde Drucksenkung um $15 \mathrm{mmHg}$ kann bei einer Vielzahl der Patienten durch Lokaltherapie nicht erreicht werden, egal mit welcher Tropfkombination. Hier müssen wir rechtzeitig operieren.

Die Autoren würden sich freuen, einen kleinen Beitrag zum besseren Verständnis der medikamentösen Glaukomtherapie geleistet zu haben.

Mein Dank gilt allen Autoren für ihre Mühen und die Bereitschaft, ihr Wissen mit uns zu teilen.

Mit den besten kollegialen Grüßen aus Köln

Ihr

Michael Diestelhorst

\section{Korrespondenzadresse}

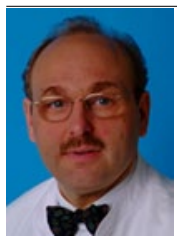

Prof. Dr. M. Diestelhorst

Klinik und Poliklinik für

allgemeine Augenheilkunde, Uniklinik Köln

Kerpener Str. 62, 50937 Köln michael.diestelhorst@ uk-koeln.de

Interessenkonflikt. M. Diestelhorst gibt an, dass kein Interessenkonflikt besteht. 
Hier steht eine Anzeige.

黛 Springer 